\title{
FOURIER FRAMES FOR THE CANTOR-4 SET
}

\author{
GABRIEL PICIOROAGA AND ERIC S. WEBER
}

\begin{abstract}
The measure supported on the Cantor- 4 set constructed by Jorgensen-Pedersen is known to have a Fourier basis, i.e. that it possess a sequence of exponentials which form an orthonormal basis. We construct Fourier frames for this measure via a dilation theory type construction. We expand the Cantor- 4 set to a 2 dimensional fractal which admits a representation of a Cuntz algebra. Using the action of this algebra, an orthonormal set is generated on the larger fractal, which is then projected onto the Cantor- 4 set to produce a Fourier frame.
\end{abstract}

Jorgensen and Pedersen [10] demonstrated that there exist singular measures $\nu$ which are spectral-that is, they possess a sequence of exponential functions which form an orthonormal basis in $L^{2}(\nu)$. The canonical example of such a singular and spectral measure is the uniform measure on the Cantor 4-set defined as follows:

$$
C_{4}=\left\{x \in[0,1]: x=\sum_{k=1}^{\infty} \frac{a_{k}}{4^{k}}, a_{k} \in\{0,2\}\right\} .
$$

This is analogous to the standard middle third Cantor set where $4^{k}$ replaces $3^{k}$. The set $C_{4}$ can also be described as the attractor set of the following iterated function system on $\mathbb{R}$ :

$$
\tau_{0}(x)=\frac{x}{4}, \quad \tau_{2}(x)=\frac{x+2}{4} .
$$

The uniform measure on the set $C_{4}$ then is the unique probability measure $\mu_{4}$ which is invariant under this iterated function system:

$$
\int f(x) d \mu_{4}(x)=\frac{1}{2}\left(\int f\left(\tau_{0}(x)\right) d \mu_{4}(x)+\int f\left(\tau_{2}(x)\right) d \mu_{4}(x)\right)
$$

for all $f \in C(\mathbb{R})$, see [9] for details. The standard spectrum for $\mu_{4}$ is $\Gamma_{4}=\left\{\sum_{n=0}^{N} l_{n} 4^{n}: l_{n} \in\right.$ $\{0,1\}\}$, though there are many spectra [4, 2].

Remarkably, Jorgensen and Pedersen prove that the uniform measure $\mu_{3}$ on the standard middle third Cantor set is not spectral. Indeed, there are no three mutually orthogonal exponentials in $L^{2}\left(\mu_{3}\right)$. Thus, there has been much attention on whether there exists a Fourier frame for $L^{2}\left(\mu_{3}\right)$-the problem is still unresolved, but see [5, 6] for progress in this regard. In this paper, we will construct Fourier frames for $L^{2}\left(\mu_{4}\right)$ using a dilation theory type argument. The motivation is whether the construction we demonstrate here for $\mu_{4}$ will be applicable to $\mu_{3}$. Fourier frames for $\mu_{4}$ were constructed in [6] using a duality type construction.

Date: July 13, 2018.

2010 Mathematics Subject Classification. Primary: 42C15, 28A80; Secondary 42B05, 46L89.

Key words and phrases. Fourier series, frames, fractals, iterated function system, Cuntz algebra. 
A frame for a Hilbert space $H$ is a sequence $\left\{x_{n}\right\}_{n \in I} \subset H$ such that there exists constants $A, B>0$ such that for all $v \in H$,

$$
A\|v\|^{2} \leq \sum_{n \in I}\left|\left\langle v, x_{n}\right\rangle\right|^{2} \leq B\|v\|^{2}
$$

The largest $A$ and smallest $B$ which satisfy these inequalities are called the frame bounds. The frame is called a Parseval frame if both frame bounds are 1 . The sequence $\left\{x_{n}\right\}_{n \in I}$ is a Bessel sequence if there exists a constant $B$ which satisfies the second inequality, whether or not the first inequality holds; $B$ is called the Bessel bound. A Fourier frame for $L^{2}\left(\mu_{4}\right)$ is a sequence of frequencies $\left\{\lambda_{n}\right\}_{n \in I} \subset \mathbb{R}$ together with a sequence of "weights" $\left\{d_{n}\right\}_{n \in I} \subset \mathbb{C}$ such that $x_{n}=d_{n} e^{2 \pi i \lambda_{n} x}$ is a frame. Fourier frames (unweighted) for Lebesgue measure were introduced by Duffin and Schaffer [3], see also Ortega-Cerda and Seip [13].

It was proven in [8] that a frame for a Hilbert space can be dilated to a Riesz basis for a bigger space, that is to say, that any frame is the image under a projection of a Riesz basis. Moreover, a Parseval frame is the image of an orthonormal basis under a projection. This result is now known to be a consequence of the Naimark dilation theory. This will be our recipe for constructing a Fourier frame: constructing a basis in a bigger space and then projecting onto a subspace. We require the following result along these lines [1]:

Lemma 1. Let $H$ be a Hilbert space, $V, K$ closed subspaces, and let $P_{V}$ be the projection onto $V$. If $\left\{x_{n}\right\}_{n \in I}$ is a frame in $K$ with frame bounds $A, B$, then:

1. $\left\{P_{V} x_{n}\right\}_{n \in I}$ is a Bessel sequence in $V$ with Bessel bound no greater than $B$;

2. if the projection $P_{V}: K \rightarrow V$ is onto, then $\left\{P_{V} x_{n}\right\}_{n \in I}$ is a frame in $V$;

3. if $V \subset K$, then then $\left\{P_{V} x_{n}\right\}_{n \in I}$ is a frame in $V$ with frame bounds between $A$ and $B$.

Note that if $V \subset K$ and $\left\{x_{n}\right\}_{n \in I}$ is a Parseval frame for $K$, then $\left\{P_{V} x_{n}\right\}_{n \in I}$ is a Parseval frame for $V$. In the second item above, it is possible that the lower frame bound for $\left\{P_{V} x_{n}\right\}$ is smaller than $A$, but the upper frame bound is still no greater than $B$.

The foundation of our construction is a dilation theory type argument. Our first step, described in Section 1, is to consider the fractal like set $C_{4} \times[0,1]$, which we will view in terms of an iterated function system. This IFS will give rise to a representation of the Cuntz algebra $\mathcal{O}_{4}$ on $L^{2}\left(\mu_{4} \times \lambda\right)$ since $\mu_{4} \times \lambda$ is the invariant measure under the IFS. Then in Section 2, we will generate via the action of $\mathcal{O}_{4}$ an orthonormal set in $L^{2}\left(\mu_{4} \times \lambda\right)$ whose vectors have a particular structure. In Section [3, we consider a subspace $V$ of $L^{2}\left(\mu_{4} \times \lambda\right)$ which can be naturally identified with $L^{2}\left(\mu_{4}\right)$, and then project the orthonormal set onto $V$ to, ultimately, obtain a frame. Of paramount importance will be whether the orthonormal set generated by $\mathcal{O}_{4}$ spans the subspace $V$ so that the projection yields a Parseval frame. Section 4 demonstrates concrete constructions in which this occurs, and identifies all possible Fourier frames that can be constructed using this method.

We note here that there may be Fourier frames for $L^{2}\left(\mu_{4}\right)$ which cannot be constructed in this manner, but we are unaware of such an example.

\section{Dilation of the Cantor-4 Set}

We wish to construct a Hilbert space $H$ which contains $L^{2}\left(\mu_{4}\right)$ as a subspace in a natural way. We will do this by making the fractal $C_{4}$ bigger as follows. We begin with an iterated 
function system on $\mathbb{R}^{2}$ given by:

$$
\Upsilon_{0}(x, y)=\left(\frac{x}{4}, \frac{y}{2}\right), \Upsilon_{1}(x, y)=\left(\frac{x+2}{4}, \frac{y}{2}\right), \Upsilon_{2}(x, y)=\left(\frac{x}{4}, \frac{y+1}{2}\right), \Upsilon_{3}(x, y)=\left(\frac{x+2}{4}, \frac{y+1}{2}\right) .
$$

As these are contractions on $\mathbb{R}^{2}$, there exists a compact attractor set, which is readily verified to be $C_{4} \times[0,1]$. Likewise, by Hutchinson [9], there exists an invariant probability measure supported on $C_{4} \times[0,1]$; it is readily verified that this invariant measure is $\mu_{4} \times \lambda$, where $\lambda$ denotes the Lebesgue measure restricted to $[0,1]$. Thus, for every continuous function $f: \mathbb{R}^{2} \rightarrow \mathbb{C}$

$$
\begin{aligned}
\int f(x, y) d\left(\mu_{4} \times \lambda\right)=\frac{1}{4} & \left(\int f\left(\frac{x}{4}, \frac{y}{2}\right) d\left(\mu_{4} \times \lambda\right)+\int f\left(\frac{x+2}{4}, \frac{y}{2}\right) d\left(\mu_{4} \times \lambda\right)\right. \\
& \left.+\int f\left(\frac{x}{4}, \frac{y+1}{2}\right) d\left(\mu_{4} \times \lambda\right)+\int f\left(\frac{x+2}{4}, \frac{y+1}{2}\right) d\left(\mu_{4} \times \lambda\right)\right) .
\end{aligned}
$$

The iterated function system $\Upsilon_{j}$ has a left inverse on $C_{4} \times[0,1]$, given by

$$
R: C_{4} \times[0,1] \rightarrow C_{4} \times[0,1]:(x, y) \mapsto(4 x, 2 y) \bmod 1,
$$

so that $R \circ \Upsilon_{j}(x, y)=(x, y)$ for $j=0,1,2,3$.

We will use the iterated function system to define an action of the Cuntz algebra $\mathcal{O}_{4}$ on $L^{2}\left(\mu_{4} \times \lambda\right)$. To do so, we choose filters

$$
\begin{aligned}
& m_{0}(x, y)=H_{0}(x, y) \\
& m_{1}(x, y)=e^{2 \pi i x} H_{1}(x, y) \\
& m_{2}(x, y)=e^{4 \pi i x} H_{2}(x, y) \\
& m_{3}(x, y)=e^{6 \pi i x} H_{3}(x, y)
\end{aligned}
$$

where

$$
H_{j}(x, y)=\sum_{k=0}^{3} a_{j k} \chi_{\Upsilon_{k}\left(C_{4} \times[0,1]\right)}(x, y)
$$

for some choice of scalar coefficients $a_{j k}$. In order to obtain a representation of $\mathcal{O}_{4}$ on $L^{2}\left(\mu_{4} \times\right.$ $\lambda$ ), we require that the above filters satisfy the matrix equation $\mathcal{M}^{*}(x, y) \mathcal{M}(x, y)=I$ for $\mu_{4} \times \lambda$ almost every $(x, y)$, where

$$
\mathcal{M}(x, y)=\left(\begin{array}{llll}
m_{0}\left(\Upsilon_{0}(x, y)\right) & m_{0}\left(\Upsilon_{1}(x, y)\right) & m_{0}\left(\Upsilon_{2}(x, y)\right) & m_{0}\left(\Upsilon_{3}(x, y)\right) \\
m_{1}\left(\Upsilon_{0}(x, y)\right) & m_{1}\left(\Upsilon_{1}(x, y)\right) & m_{1}\left(\Upsilon_{2}(x, y)\right) & m_{1}\left(\Upsilon_{3}(x, y)\right) \\
m_{2}\left(\Upsilon_{0}(x, y)\right) & m_{2}\left(\Upsilon_{1}(x, y)\right) & m_{2}\left(\Upsilon_{2}(x, y)\right) & m_{2}\left(\Upsilon_{3}(x, y)\right) \\
m_{3}\left(\Upsilon_{0}(x, y)\right) & m_{3}\left(\Upsilon_{1}(x, y)\right) & m_{3}\left(\Upsilon_{2}(x, y)\right) & m_{3}\left(\Upsilon_{3}(x, y)\right)
\end{array}\right)
$$

For our choice of filters, the matrix $\mathcal{M}$ becomes

$$
\mathcal{M}(x, y)=\left(\begin{array}{rrrr}
a_{00} & a_{01} & a_{02} & a_{03} \\
e^{\pi i x / 2} a_{10} & -e^{\pi i x / 2} a_{11} & e^{\pi i x / 2} a_{12} & -e^{\pi i x / 2} a_{13} \\
e^{\pi i x} a_{20} & e^{\pi i x} a_{21} & e^{\pi i x} a_{22} & e^{\pi i x} a_{23} \\
e^{3 \pi i x / 2} a_{30} & -e^{3 \pi i x / 2} a_{31} & e^{3 \pi i x / 2} a_{32} & -e^{3 \pi i x / 2} a_{33}
\end{array}\right)
$$


which is unitary if and only if the matrix

$$
H=\left(\begin{array}{rrrr}
a_{00} & a_{01} & a_{02} & a_{03} \\
a_{10} & -a_{11} & a_{12} & -a_{13} \\
a_{20} & a_{21} & a_{22} & a_{23} \\
a_{30} & -a_{31} & a_{32} & -a_{33}
\end{array}\right)
$$

is unitary. For the remainder of this section, we assume that $H$ is unitary.

Lemma 2. The operator $S_{j}: L^{2}\left(\mu_{4} \times \lambda\right) \rightarrow L^{2}\left(\mu_{4} \times \lambda\right)$ given by

$$
\left[S_{j} f\right](x, y)=\sqrt{4} m_{j}(x, y) f(R(x, y))
$$

is an isometry.

Proof. We calculate:

$$
\begin{aligned}
\left\|S_{j} f\right\|^{2} & =\int\left|\sqrt{4} m_{j}(x, y) f(R(x, y))\right|^{2} d\left(\mu_{4} \times \lambda\right) \\
& =\frac{1}{4} \sum_{k=0}^{3} \int 4\left|m_{j}\left(\Upsilon_{k}(x, y)\right) f\left(R\left(\Upsilon_{k}(x, y)\right)\right)\right|^{2} d\left(\mu_{4} \times \lambda\right) \\
& =\int\left(\sum_{k=0}^{3}\left|m_{j}\left(\Upsilon_{k}(x, y)\right)\right|^{2}\right)|f(x, y)|^{2} d\left(\mu_{4} \times \lambda\right)
\end{aligned}
$$

We used Equation (10) in the second line. The sum in the integral is the square of the Euclidean norm of the $j$-th row of the matrix $\mathcal{M}$, which is unitary. Hence, the sum is 1 , so the integral is $\|f\|^{2}$, as required.

Lemma 3. The adjoint is given by

$$
\left[S_{j}^{*} f\right](x, y)=\frac{1}{2} \sum_{k=0}^{3} \overline{m_{j}\left(\Upsilon_{k}(x, y)\right)} f\left(\Upsilon_{j}(x, y)\right) .
$$

Proof. Let $f, g \in L^{2}\left(\mu_{4} \times \lambda\right)$. We calculate

$$
\begin{aligned}
\left\langle S_{j} f, g\right\rangle & =\int \sqrt{4} m_{j}(x, y) f(R(x, y)) \overline{g(x, y)} d\left(\mu_{4} \times \lambda\right) \\
& =\frac{1}{4} \sum_{k=0}^{3} \int \sqrt{4} m_{j}\left(\Upsilon_{k}(x, y)\right) f\left(R\left(\Upsilon_{k}(x, y)\right)\right) \overline{g\left(\Upsilon_{k}(x, y)\right)} d\left(\mu_{4} \times \lambda\right) \\
& =\int f(x, y) \overline{\left(\frac{1}{2} \sum_{k=0}^{3} \overline{m_{j}\left(\Upsilon_{k}(x, y)\right)} g\left(\Upsilon_{k}(x, y)\right)\right)} d\left(\mu_{4} \times \lambda\right)
\end{aligned}
$$

where we use Equation (1) and the fact that $R$ is a left inverse of $\Upsilon_{k}$.

Lemma 4. The isometries $S_{j}$ satisfy the Cuntz relations:

$$
S_{j}^{*} S_{k}=\delta_{j k} I, \quad \sum_{k=0}^{3} S_{k} S_{k}^{*}=I .
$$


Proof. We consider the orthogonality relation first. Let $f \in L^{2}\left(\mu_{4} \times \lambda\right)$. We calculate:

$$
\begin{aligned}
{\left[S_{j}^{*} S_{k} f\right](x, y) } & =\frac{1}{2} \sum_{\ell=0}^{3} \overline{m_{j}\left(\Upsilon_{\ell}(x, y)\right)}\left[S_{k} f\right]\left(\Upsilon_{\ell}(x, y)\right) \\
& =\frac{1}{2} \sum_{\ell=0}^{3} \overline{m_{j}\left(\Upsilon_{\ell}(x, y)\right)} \sqrt{4} m_{k}\left(\Upsilon_{\ell}(x, y)\right) f\left(R\left(\Upsilon_{\ell}(x, y)\right)\right) \\
& =\left(\sum_{\ell=0}^{3} \overline{m_{j}\left(\Upsilon_{\ell}(x, y)\right)} m_{k}\left(\Upsilon_{\ell}(x, y)\right)\right) f(x, y)
\end{aligned}
$$

Note that the sum is the scalar product of the $k$-th row with the $j$-th row of the matrix $\mathcal{M}$, which is unitary. Hence, the sum is $\delta_{j k}$ as required.

Now for the identity relation, let $f, g \in L^{2}\left(\mu_{4} \times \lambda\right)$. We calculate:

$$
\begin{aligned}
\left\langle\sum_{k=0}^{3} S_{k} S_{k}^{*} f, g\right\rangle=\sum_{k=0}^{3}\left\langle S_{k}^{*} f, S_{k}^{*} g\right\rangle & \\
\quad= & \sum_{k=0}^{3} \int\left(\frac{1}{2} \sum_{\ell=0}^{3} \overline{m_{k}\left(\Upsilon_{\ell}(x, y)\right)} f\left(\Upsilon_{\ell}(x, y)\right)\right)\left(\overline{\frac{1}{2} \sum_{n=0}^{3} \overline{m_{k}\left(\Upsilon_{n}(x, y)\right)} g\left(\Upsilon_{n}(x, y)\right)}\right) d\left(\mu_{4} \times \lambda\right) \\
& =\sum_{\ell=0}^{3} \sum_{n=0}^{3} \frac{1}{4} \int\left(\sum_{k=0}^{3} \overline{m_{k}\left(\Upsilon_{\ell}(x, y)\right)} m_{k}\left(\Upsilon_{n}(x, y)\right)\right) f\left(\Upsilon_{\ell}(x, y)\right) \overline{g\left(\Upsilon_{n}(x, y)\right)} d\left(\mu_{4} \times \lambda\right) \\
& =\frac{1}{4} \sum_{n=0}^{3} \int f\left(\Upsilon_{n}(x, y)\right) \overline{g\left(\Upsilon_{n}(x, y)\right)} d\left(\mu_{4} \times \lambda\right) \\
& =\int f(x, y) \overline{g(x, y)} d\left(\mu_{4} \times \lambda\right) \\
& =\langle f, g\rangle .
\end{aligned}
$$

Note that the sum over $k$ in the third line is the scalar product of the $\ell$-th column with the $n$-th column of $\mathcal{M}$, so the sum collapses to $\delta_{\ell n}$. The sum on $n$ in the fourth line collapses by Equation (11).

\section{Orthonormal Sets in $L^{2}\left(\mu_{4} \times \lambda\right)$}

Since the isometries $S_{j}$ satisfy the Cuntz relations, we can use them to generate orthonormal sets in the space $L^{2}\left(\mu_{4} \times \lambda\right)$. We do so by having the isometries act on a generating vector. We consider words in the alphabet $\{0,1,2,3\}$; let $W_{4}$ denote the set of all such words. For a word $\omega=j_{K} j_{K-1} \ldots j_{1}$, we denote by $|\omega|=K$ the length of the word, and define

$$
S_{\omega} f=S_{j_{K}} S_{j_{K-1}} \ldots S_{j_{1}} f .
$$

Definition 1. Let

$$
X_{4}=\left\{\omega \in W_{4}:|\omega|=1\right\} \cup\left\{\omega \in W_{4}:|\omega| \geq 2, j_{1} \neq 0\right\} .
$$

For convenience, we allow the empty word $\omega_{\emptyset}$ with length 0 , and define $S_{\omega_{\emptyset}}=I$, the identity. 
Lemma 5. Suppose $f \in L^{2}\left(\mu_{4} \times \lambda\right)$ with $\|f\|=1$, and that $S_{0} f=f$. Then,

$$
\left\{S_{\omega} f: \omega \in X_{4}\right\}
$$

is an orthonormal set.

Proof. Suppose $\omega, \omega^{\prime} \in X_{4}$ with $\omega \neq \omega^{\prime}$. First consider $|\omega|=\left|\omega^{\prime}\right|$, with $\omega=j_{K} \ldots j_{1}$ and $\omega^{\prime}=i_{K} \ldots i_{1}$. Suppose that $\ell$ is the largest index such that $j_{\ell} \neq i_{\ell}$. Then we have

$$
\left\langle S_{\omega} f, S_{\omega^{\prime}} f\right\rangle=\left\langle S_{j_{\ell}} \ldots S_{j_{1}} f, S_{i_{\ell}} \ldots S_{i_{1}} f\right\rangle=\left\langle S_{i_{\ell}}^{*} S_{j_{\ell}} \ldots S_{j_{1}} f, S_{i_{\ell-1}} \ldots S_{i_{1}} f\right\rangle=0
$$

by the orthogonality condition of the Cuntz relations.

Now, if $K=|\omega|>\left|\omega^{\prime}\right|=M$, with $\omega^{\prime}=i_{M} \ldots i_{1}$, we define the word $\rho=i_{M} \ldots i_{1} 0 \ldots 0$ so that $|\rho|=K$. Note that $\rho \notin X_{4}$ so $\omega \neq \rho$. Note further that $S_{\omega^{\prime}} f=S_{\rho} f$. Thus, by a similar argument to that above, we have

$$
\left\langle S_{\omega} f, S_{\omega^{\prime}} f\right\rangle=0
$$

Remark 1. The set $\left\{S_{\omega} f: \omega \in X_{4}\right\}$ need not be complete. We will provide an example of this in Example 1 in Section 4 .

Our goal is to project the set $\left\{S_{\omega} f: \omega \in X_{4}\right\}$ onto some subspace $V$ of $L^{2}\left(\mu_{4} \times \lambda\right)$ to obtain a frame. To that end, we need to know when the projection $\left\{P_{V} S_{\omega} f: \omega \in X_{4}\right\}$ is a frame, which by Lemma 1 requires the projection $P_{V}: K \rightarrow V$ to be onto, where $K$ is the subspace spanned by $\left\{S_{\omega} f: \omega \in X_{4}\right\}$. The tool we will use is the following result, which is a minor adaptation of a result from [7]; we will not use this result directly, but will use all of the critical components.

Theorem 1. Let $\mathcal{H}$ be a Hilbert space, $\mathcal{K} \subset \mathcal{H}$ a closed subspace, and $\left(S_{i}\right)_{i=0}^{N-1}$ be a representation of the Cuntz algebra $\mathcal{O}_{N}$. Let $\mathcal{E}$ be an orthonormal set in $\mathcal{H}$ and $f: X \rightarrow \mathcal{K}$ a norm continuous function on a topological space $X$ with the following properties:

i) $\mathcal{E}=\cup_{i=0}^{N-1} S_{i} \mathcal{E}$ where the union is disjoint.

ii) $\overline{\operatorname{span}}\{f(t): t \in X\}=\mathcal{K}$ and $\|f(t)\|=1$, for all $t \in X$.

iii) There exist functions $\mathfrak{m}_{i}: X \rightarrow \mathbb{C}, g_{i}: X \rightarrow X, i=0, \ldots, N-1$ such that

$$
S_{i}^{*} f(t)=\mathfrak{m}_{i}(t) f\left(g_{i}(t)\right), \quad t \in X .
$$

iv) There exist $c_{0} \in X$ such that $f\left(c_{0}\right) \in \overline{\operatorname{span}} \mathcal{E}$.

v) The only function $h \in \mathcal{C}(X)$ with $h \geq 0, h(c)=1, \forall c \in\{x \in X: f(x) \in \overline{\text { span }} \mathcal{E}\}$, and

$$
h(t)=\sum_{i=0}^{N-1}\left|\mathfrak{m}_{i}(t)\right|^{2} h\left(g_{i}(t)\right), \quad t \in X
$$

are the constant functions.

Then $\mathcal{K} \subset \overline{\operatorname{span} \mathcal{E}}$.

\section{The Projection}

Recall the definition of the filters $m_{j}(x, y)=e^{2 \pi i j x} H_{j}(x, y)$ from Section 1, We choose the filter coefficients $a_{j k}$ so that the matrix $H$ is unitary. We place the additional constraint that

$$
a_{00}=a_{01}=a_{02}=a_{03}=\frac{1}{2}
$$


so that $S_{0} \mathbb{1}=\mathbb{1}$, where $\mathbb{1}$ the function in $L^{2}\left(\mu_{4} \times \lambda\right)$ which is identically 1 . As $S_{0} \mathbb{1}=\mathbb{1}$, by Lemma 5 , the set $\left\{S_{\omega} \mathbb{1}: \omega \in X_{4}\right\}$ is orthonormal. Moreover, we place the additional constraint that for every $j, a_{j 0}+a_{j 2}=a_{j 1}+a_{j 3}$, which will be required for our calculation of the projection.

Definition 2. We define the subspace $V=\left\{f \in L^{2}\left(\mu_{4} \times \lambda\right): f(x, y)=g(x) \chi_{[0,1]}(y), g \in\right.$ $\left.L^{2}\left(\mu_{4}\right)\right\}$. Note that the subspace $V$ can be identified with $L^{2}\left(\mu_{4}\right)$ via the isometric isomorphism $g \mapsto g(x) \chi_{[0,1]}(y)$. We will suppress the $y$ variable in the future.

Definition 3. We define a function $c: X_{4} \rightarrow \mathbb{N}_{0}$ as follows: for a word $\omega=j_{K} j_{K-1} \ldots j_{1}$,

$$
c(\omega)=\sum_{k=1}^{K} j_{k} 4^{K-k} .
$$

Here $\mathbb{N}_{0}=\mathbb{N} \cup\{0\}$. It is readily verified that $c$ is a bijection.

Lemma 6. For a word $\omega=j_{K} j_{K-1} \ldots j_{1}$,

$$
S_{\omega} \mathbb{1}=e^{2 \pi i c(\omega) x}\left(\prod_{k=1}^{K} 2 H_{j_{k}}\left(R^{K-k}(x, y)\right)\right) .
$$

Proof. We proceed by induction on the length of the word $\omega$. The equality is readily verified for $|\omega|=1$. Let $\omega_{0}=j_{K-1} j_{n-2} \ldots j_{1}$. We have

$$
\begin{aligned}
S_{\omega} \mathbb{1} & =S_{j_{K}} S_{\omega_{0}} \mathbb{1} \\
& =S_{j_{K}}\left[e^{2 \pi i c\left(\omega_{0}\right) x}\left(\prod_{k=1}^{K-1} 2 H_{j_{k}}\left(R^{K-1-k}(x, y)\right)\right)\right] \\
& =2 e^{2 \pi i \lambda_{j_{K}} x} H_{j_{K}}(x, y) e^{2 \pi i c\left(\omega_{0}\right) \cdot 4 x}\left(\prod_{k=1}^{K-1} H_{j_{k}}\left(R^{K-k}(x, y)\right)\right) \\
& =2 e^{2 \pi i\left(\lambda_{j_{K}}+4 c\left(\omega_{0}\right)\right) x} H_{j_{K}}\left(R^{K-K}(x, y)\right)\left(\prod_{k=1}^{K-1} 2 H_{j_{k}}\left(R^{K-k}(x, y)\right)\right) \\
& =e^{2 \pi i c(\omega) x}\left(\prod_{k=1}^{K} 2 H_{j_{k}}\left(R^{K-k}(x, y)\right)\right) .
\end{aligned}
$$

The last line above is justified by the following calculation:

$$
\begin{aligned}
\lambda_{j_{K}}+4 c\left(\omega_{0}\right) & =\lambda_{j_{K}}+4\left(\sum_{k=1}^{K-1} \lambda_{j_{k}} 4^{K-1-k}\right) \\
& =\lambda_{j_{K}} 4^{K-K}+\sum_{k=1}^{K-1} \lambda_{j_{k}} 4^{K-k} \\
& =\sum_{k=1}^{K} \lambda_{j_{k}} 4^{K-k} \\
& =c(\omega) .
\end{aligned}
$$


We wish to project the vectors $S_{\omega} \mathbb{1}$ onto the subspace $V$. The following lemma calculates that projection, where $P_{V}$ denotes the projection onto the subspace $V$.

Lemma 7. If $f(x, y)=g(x) h(x, y)$ with $g \in L^{2}\left(\mu_{4}\right)$ and $h \in L^{\infty}\left(\mu_{4} \times \lambda\right)$, then

$$
\left[P_{V} f\right](x, y)=g(x) G(x)
$$

where $G(x)=\int_{[0,1]} h(x, y) d \lambda(y)$.

Proof. We verify that for every $F(x) \in L^{2}\left(\mu_{4}\right), f(x, y)-g(x) G(x)$ is orthogonal to $F(x)$. We calculate utilizing Fubini's theorem:

$$
\begin{aligned}
\langle f-g G, F\rangle & =\iint g(x) h(x, y) \overline{F(x)} d\left(\mu_{4} \times \lambda\right)-\iint g(x) G(x) \overline{F(x)} d\left(\mu_{4} \times \lambda\right) \\
& =\int_{C_{4}} g(x) \overline{F(x)}\left(\int_{[0,1]} h(x, y)-G(x) d \lambda(y)\right) d \mu_{4}(x) \\
& =\int_{C_{4}} g(x) \overline{F(x)}(G(x)-G(x)) d \mu_{4}(x) \\
& =0 .
\end{aligned}
$$

For the purposes of the following lemma, $\alpha x$ and $\beta y$ are understood to be modulo 1 .

Lemma 8. For any word $\omega=j_{K} j_{K-1} \ldots j_{1}$,

$$
\int \prod_{k=1}^{K} 2 H_{j_{k}}\left(R^{k-1}(x, y)\right) d \lambda(y)=\prod_{k=1}^{K} 2 \int H_{j_{k}}\left(4^{k-1} x, y\right) d \lambda(y) .
$$

Proof. Let $F_{m}(x, y)=\prod_{k=m}^{K} 2 H_{j_{k}}\left(4^{k-1} x, 2^{k-m} y\right)$. Note that

$$
F_{m}\left(x, \frac{y}{2}\right)=2 H_{j_{m}}\left(4^{m-1} x, \frac{y}{2}\right)\left(\prod_{k=m+1}^{K} 2 H_{j_{k}}\left(4^{k-1} x, 2^{k-(m+1)} y\right)\right)=2 H_{j_{m}}\left(4^{m-1} x, \frac{y}{2}\right) F_{m+1}(x, y) .
$$

Likewise for $F_{m}\left(x, \frac{y+1}{2}\right)$.

Since $\lambda$ is the invariant measure for the iterated function system $y \mapsto \frac{y}{2}, y \mapsto \frac{y+1}{2}$, we calculate:

$$
\begin{aligned}
\int_{0}^{1} F_{m}(x, y) d \lambda(y) & =\frac{1}{2}\left[\int_{0}^{1} F_{m}\left(x, \frac{y}{2}\right) d \lambda(y)+\int_{0}^{1} F_{m}\left(x, \frac{y+1}{2}\right) d \lambda(y)\right] \\
& =\frac{1}{2}\left[\int_{0}^{1} 2 H_{j_{m}}\left(4^{m-1} x, \frac{y}{2}\right) F_{m+1}(x, y)+2 H_{j_{m}}\left(4^{m-1} x, \frac{y+1}{2}\right) F_{m+1}(x, y) d \lambda(y)\right] \\
& =\frac{1}{2}\left[\int_{0}^{1} 2 a_{j_{m}, q} F_{m+1}(x, y)+2 a_{j_{m}, q+2} F_{m+1}(x, y) d \lambda(y)\right] \\
& =\frac{1}{2}\left[2 a_{j_{m}, q}+2 a_{j_{m}, q+2}\right] \cdot\left[\int_{0}^{1} F_{m+1}(x, y) d \lambda(y)\right] \\
& =\left[\int_{0}^{1} 2 H_{j_{m}}\left(4^{m-1} x, y\right) d \lambda(y)\right] \cdot\left[\int_{0}^{1} F_{m+1}(x, y) d \lambda(y)\right]
\end{aligned}
$$

where $q=0$ if $0 \leq 4^{m-1} x<\frac{1}{2}$, and $q=1$ if $\frac{1}{2} \leq 4^{m-1} x<1$. 
The result now follows by a standard induction argument.

Proposition 1. Suppose the filters $m_{j}(x, y)$ are chosen so that

i) the matrix $H$ is unitary,

ii) $a_{00}=a_{01}=a_{02}=a_{03}=\frac{1}{2}$, and

iii) for $j=0,1,2,3, a_{j 0}+a_{j 2}=a_{j 1}+a_{j 3}$.

Then for any word $\omega=j_{K} \ldots j_{1}$,

$$
P_{V} S_{\omega} \mathbb{1}=d_{\omega} e^{2 \pi i c(\omega) x}
$$

where

$$
d_{\omega}=\prod_{k=1}^{K}\left(a_{j_{k} 0}+a_{j_{k} 2}\right) .
$$

Proof. We apply the previous three Lemmas to obtain

$$
\begin{aligned}
{\left[P_{V} S_{\omega} \mathbb{1}\right](x, y) } & =e^{2 \pi i c(\omega) x} \int \prod_{k=1}^{K} 2 H_{j_{k}}\left(4^{k-1} x, y\right) d \lambda(y) \\
& =e^{2 \pi i c(\omega) x} \prod_{k=1}^{K} 2 \int H_{j_{k}}\left(4^{k-1} x, y\right) d \lambda(y)
\end{aligned}
$$

By assumption iii), the integral $\int H_{j_{k}}\left(4^{k-1} x, y\right) d \lambda(y)$ is independent of $x$, and the value of the integral is $\frac{a_{j 0}}{2}+\frac{a_{j 2}}{2}$. Equation 4 now follows.

\section{Concrete Constructions}

We now turn to concrete constructions of Fourier frames for $\mu_{4}$. The hypotheses of Lemma 5 and Proposition 1 require $H$ to be unitary and requires the matrix

$$
A=\left(\begin{array}{cccc}
a_{00} & a_{01} & a_{02} & a_{03} \\
a_{10} & a_{11} & a_{12} & a_{13} \\
a_{20} & a_{21} & a_{22} & a_{23} \\
a_{30} & a_{31} & a_{32} & a_{33}
\end{array}\right)
$$

to have the first row be identically $\frac{1}{2}$ and to have the vector $\left(\begin{array}{llll}1 & -1 & 1 & -1\end{array}\right)^{T}$ in the kernel.

We can use Hadamard matrices to construct examples of such a matrix $A$. Every $4 \times 4$ Hadamard matrix is a permutation of the following matrix:

$$
U_{\rho}=\frac{1}{2}\left(\begin{array}{rrrr}
1 & 1 & 1 & 1 \\
1 & -1 & \rho & -\rho \\
1 & 1 & -1 & -1 \\
1 & -1 & -\rho & \rho
\end{array}\right)
$$

where $\rho$ is any complex number of modulus 1 .

If we set $H=U_{\rho}$, we obtain

$$
A=\frac{1}{2}\left(\begin{array}{rrrr}
1 & 1 & 1 & 1 \\
1 & 1 & \rho & \rho \\
1 & 1 & -1 & -1 \\
1 & 1 & -\rho & -\rho
\end{array}\right)
$$


which has the requisite properties to apply Lemma 5 and Proposition 1.

We define for $k=1,2,3, l_{k}: \mathbb{N}_{0} \rightarrow \mathbb{N}_{0}$ by $l_{k}(n)$ is the number of digits equal to $k$ in the base 4 expansion of $n$. Note that $l_{k}(0)=0$, and we follow the convention that $0^{0}=1$.

Theorem 2. For the choice $A$ as in Equation (5) with $\rho \neq-1$, the sequence

$$
\left\{\left(\frac{1+\rho}{2}\right)^{l_{1}(n)} 0^{l_{2}(n)}\left(\frac{1-\rho}{2}\right)^{l_{3}(n)} e^{2 \pi i n x}: n \in \mathbb{N}_{0}\right\}
$$

is a Parseval frame in $L^{2}\left(\mu_{4}\right)$.

Proof. By Lemma 5, we have that $\left\{S_{\omega} \mathbb{1}: \omega \in X_{4}\right\}$ is an orthonormal set. For a word $\omega=j_{K} j_{K-1} \ldots j_{1}$, Proposition 1 yields that

$$
P_{V} S_{\omega} \mathbb{1}=e^{2 \pi i c(\omega) x} \prod_{k=1}^{K}\left(a_{j_{k} 0}+a_{j_{k} 2}\right) .
$$

Then, setting $n=c(\omega)$, we obtain

$$
P_{V} S_{\omega} \mathbb{1}=e^{2 \pi i n x}\left(a_{00}+a_{02}\right)^{K-l_{1}(n)-l_{2}(n)-l_{3}(n)} \prod_{j=1}^{3}\left(a_{j 0}+a_{j 2}\right)^{l_{j}(n)} .
$$

Since

$$
a_{00}+a_{02}=1, \quad a_{10}+a_{12}=\frac{1+\rho}{2}, \quad a_{20}+a_{22}=0, \quad a_{30}+a_{32}=\frac{1-\rho}{2},
$$

it follows that

$$
P_{V} S_{\omega} \mathbb{1}=\left(\frac{1+\rho}{2}\right)^{l_{1}(n)} 0^{l_{2}(n)}\left(\frac{1-\rho}{2}\right)^{l_{3}(n)} e^{2 \pi i n x} .
$$

Since $c$ is a bijection, the set $\left\{P_{V} S_{\omega} \mathbb{1}: \omega \in X_{4}\right\}$ coincides with the set in (6).

In order to establish that the set (6) is a Parseval frame, we wish to apply Lemma 1, which requires that the subspace $V$ is contained in the closed span of $\left\{S_{\omega} \mathbb{1}: \omega \in X_{4}\right\}$. Denote the closed span by $\mathcal{K}$. We will proceed in a manner similar to Theorem 1 . Define the function $f: \mathbb{R} \rightarrow V$ by $f(t)=e_{t}$ where $e_{t}(x, y)=e^{2 \pi i x t}$. Note that $f(0)=\mathbb{1} \in \mathcal{K}$. Likewise, define a function $h_{X}: \mathbb{R} \rightarrow \mathbb{R}$ by

$$
h_{X}(t)=\sum_{\omega \in X_{4}}\left|\left\langle f(t), S_{\omega} \mathbb{1}\right\rangle\right|^{2}=\left\|P_{\mathcal{K}} f(t)\right\|^{2}
$$

Claim 1. We have $h_{X} \equiv 1$.

Assuming for the moment that the claim holds, we deduce that $f(t) \in \mathcal{K}$ for every $t \in \mathbb{R}$. Since $\left\{f(\gamma): \gamma \in \Gamma_{4}\right\}$ is an orthonormal basis for $V$, it follows that the closed span of $\{f(t)$ : $t \in \mathbb{R}\}$ is all of $V$. We conclude that $V \subset \mathcal{K}$, and so Lemma 1 implies that $\left\{P_{V} S_{\omega} \mathbb{1}: \omega \in X_{4}\right\}$ is a Parseval frame for $V$, from which the Theorem follows.

Thus, we turn to the proof of Claim 1, First, we require $\left\{S_{\omega} \mathbb{1}: \omega \in X_{4}\right\}=\cup_{j=0}^{3}\left\{S_{j} S_{\omega} \mathbb{1}\right.$ : $\left.\omega \in X_{4}\right\}$, where the union is disjoint. Clearly, the RHS is a subset of the LHS, and the union is disjoint. Consider an element of the LHS: $S_{\omega} \mathbb{1}$. If $|\omega| \geq 2$, we write $S_{\omega} \mathbb{1}=S_{j} S_{\omega_{0}} \mathbb{1}$ for some $j$ and some $\omega_{0} \in X_{4}$, whence $S_{\omega} \mathbb{1}$ is in the RHS. If $|\omega|=1$, then we write $S_{\omega} \mathbb{1}=S_{j} \mathbb{1}=S_{j} S_{0} \mathbb{1}$, which is again an element of the RHS. Equality now follows. 
As a consequence,

$$
\begin{aligned}
h_{X}(t) & =\sum_{\omega \in X_{4}}\left|\left\langle f(t), S_{\omega} \mathbb{1}\right\rangle\right|^{2} \\
& =\sum_{j=0}^{3} \sum_{\omega \in X_{4}}\left|\left\langle f(t), S_{j} S_{\omega} \mathbb{1}\right\rangle\right|^{2} \\
& =\sum_{j=0}^{3} \sum_{\omega \in X_{4}}\left|\left\langle S_{j}^{*} f(t), S_{\omega} \mathbb{1}\right\rangle\right|^{2} .
\end{aligned}
$$

We calculate:

$$
\begin{aligned}
{\left[S_{j}^{*} f(t)\right](x, y)=} & \frac{1}{2} \sum_{k=0}^{3} \overline{m_{j}\left(\Upsilon_{k}(x, y)\right)} e_{t}\left(\Upsilon_{k}(x, y)\right) \\
= & \frac{1}{2}\left[\overline{a_{j 0}} e^{-2 \pi i j x / 4} e_{t}\left(\frac{x}{4}, \frac{y}{2}\right)+e^{-\pi i j} \overline{a_{j 1}} e^{-2 \pi i j x / 4} e_{t}\left(\frac{x+2}{4}, \frac{y}{2}\right)\right. \\
& \left.\quad+\overline{a_{j 2}} e^{-2 \pi i j x / 4} e_{t}\left(\frac{x}{4}, \frac{y+1}{2}\right)+e^{-\pi i j} \overline{a_{j 3}} e^{-2 \pi i j x / 4} e_{t}\left(\frac{x+2}{4}, \frac{y+1}{2}\right)\right] \\
= & \frac{1}{2}\left[\overline{a_{j 0}} e^{-2 \pi i j x / 4} e_{t}\left(\frac{x}{4}, \frac{y}{2}\right)+e^{-\pi i j} \overline{a_{j 1}} e^{-2 \pi i j x / 4} e^{\pi i t} e_{t}\left(\frac{x}{4}, \frac{y}{2}\right)\right. \\
& \left.\quad+\overline{a_{j 2}} e^{-2 \pi i j x / 4} e_{t}\left(\frac{x}{4}, \frac{y}{2}\right)+e^{-\pi i j} \overline{a_{j 3}} e^{-2 \pi i j x / 4} e^{\pi i t} e_{t}\left(\frac{x}{4}, \frac{y}{2}\right)\right] \\
= & \frac{1}{2}\left[\overline{a_{j 0}}+e^{-\pi i j} \overline{a_{j 1}} e^{\pi i t}+\overline{a_{j 2}}+e^{-\pi i j} \overline{a_{j 3}} e^{\pi i t}\right] e^{-2 \pi i j x / 4} e_{t}\left(\frac{x}{4}, \frac{y}{2}\right) \\
= & \frac{1}{2}\left[\overline{a_{j 0}}+e^{-\pi i j} \overline{a_{j 1}} e^{\pi i t}+\overline{a_{j 2}}+e^{-\pi i j} \overline{a_{j 3}} e^{\pi i t}\right] e^{2 \pi i\left(t \frac{x}{4}-j \frac{x}{4}\right)} \\
= & \frac{1}{2}\left[\overline{a_{j 0}}+e^{-\pi i j} \overline{a_{j 1}} e^{\pi i t}+\overline{a_{j 2}}+e^{-\pi i j} \overline{a_{j 3}} e^{\pi i t}\right] e^{2 \pi i\left(\frac{t-j}{4} x\right)} \\
= & \frac{1}{2}\left[\overline{a_{j 0}}+e^{-\pi i j} \overline{a_{j 1}} e^{\pi i t}+\overline{a_{j 2}}+e^{-\pi i j} \overline{a_{j 3}} e^{\pi i t}\right] e_{\frac{t-j}{4}}(x, y) .
\end{aligned}
$$

Thus, we define

$$
\mathfrak{m}_{j}(t)=\frac{1}{2}\left(\overline{a_{j 0}}+\overline{a_{j 2}}\right)+\frac{e^{-\pi i j}}{2}\left(\overline{a_{j 1}}+\overline{a_{j 3}}\right) e^{\pi i t},
$$

and

$$
g_{j}(t)=\frac{t-j}{4}
$$


As a consequence, we obtain

$$
\begin{aligned}
h_{X}(t) & =\sum_{j=0}^{3} \sum_{\omega \in X_{4}}\left|\left\langle S_{j}^{*} f(t), S_{\omega} \mathbb{1}\right\rangle\right|^{2} \\
& =\sum_{j=0}^{3} \sum_{\omega \in X_{4}}\left|\left\langle\mathfrak{m}_{j}(t) f\left(g_{j}(t)\right), S_{\omega} \mathbb{1}\right\rangle\right|^{2} \\
& =\sum_{j=0}^{3}\left|\mathfrak{m}_{j}(t)\right|^{2} h_{X}\left(g_{j}(t)\right) .
\end{aligned}
$$

Because of our choice of coefficients in the matrix $A$, which has the vector $\left(\begin{array}{llll}1 & -1 & 1 & -1\end{array}\right)^{T}$ in the kernel, we have for every $j: a_{j 0}+a_{j 2}=a_{j 1}+a_{j 3}$. Thus, if we let $b_{j}=\overline{a_{j 0}}+\overline{a_{j 2}}$, the functions $\mathfrak{m}_{j}$ simplify to

for $j=0,2$, and

$$
\mathfrak{m}_{j}(t)=b_{j} e^{\pi i \frac{t}{2}} \cos \left(\pi \frac{t}{2}\right)
$$

$$
\mathfrak{m}_{j}(t)=-i b_{j} e^{\pi i \frac{t}{2}} \sin \left(\pi \frac{t}{2}\right)
$$

for $j=1,3$. Substituting these into Equation (7),

$h_{X}(t)=\cos ^{2}\left(\frac{\pi t}{2}\right) h_{X}\left(\frac{t}{4}\right)+\sin ^{2}\left(\frac{\pi t}{2}\right) \frac{|1+\bar{\rho}|^{2}}{2} h_{X}\left(\frac{t-1}{4}\right)+\sin ^{2}\left(\frac{\pi t}{2}\right) \frac{|1-\bar{\rho}|^{2}}{2} h_{X}\left(\frac{t-3}{4}\right)$.

Claim 2. The function $h_{X}$ can be extended to an entire function.

Assume for the moment that Claim 2 holds, we finish the proof of Claim 11. If $h_{X}(t)=1$ for $t \in[-1,0]$, then $h_{X}(z)=1$ for all $z \in \mathbb{C}$, and Claim 1 holds.

Now, assume to the contrary that $h_{X}(t)$ is not identically 1 on $[-1,0]$. Since $0 \leq h_{X}(t) \leq 1$ for $t$ real, then $\beta=\min \left\{h_{X}(t): t \in[-1,0]\right\}<1$. Because constant functions satisfy (8), $h_{1}:=h_{X}-\beta$ also satisfies Equation (8). There exists $t_{0}$ such that $h_{1}\left(t_{0}\right)=0$ and $t_{0} \neq 0$ as $h_{X}(0)=1$. Since $h_{1} \geq 0$ each of the terms in (8) must vanish :

$$
\begin{gathered}
\cos ^{2}\left(\frac{\pi t_{0}}{2}\right) h_{1}\left(\frac{t_{0}}{4}\right)=0 \\
\sin ^{2}\left(\frac{\pi t_{0}}{2}\right) \frac{|1+\bar{\rho}|^{2}}{2} h_{1}\left(\frac{t_{0}-1}{4}\right)=0 \\
\sin ^{2}\left(\frac{\pi t_{0}}{2}\right) \frac{|1-\bar{\rho}|^{2}}{2} h_{1}\left(\frac{t_{0}-3}{4}\right)=0
\end{gathered}
$$

Our hypothesis is that $\rho \neq-1$, so in Equation (10), the coefficient $\frac{|1+\bar{\rho}|}{2} \neq 0$.

Case 1: If $t_{0} \neq-1$ then Equation (91) implies $h_{1}\left(t_{0} / 4\right)=0=h_{1}\left(g_{0}\left(t_{0}\right)\right)$. Let $t_{1}:=g_{0}\left(t_{0}\right) \in$ $(-1,0)$; iterating the previous argument implies that $h_{1}\left(g_{0}\left(t_{1}\right)\right)=0$. Thus, we obtain an infinite sequence of zeroes of $h_{1}$.

Case 2: If $t_{0}=-1$, then the previous argument does not hold. However, we can construct another zero of $h_{1}, t_{0}^{\prime} \in(-1,0)$ to which the previous argument will hold. Indeed, if $t_{0}=-1$, 
Equation (10) implies $h_{1}\left(\left(t_{0}-1\right) / 4\right)=h_{1}(-1 / 2)=0$. Let $t_{0}^{\prime}=-1 / 2$ and continue as in Case 1.

In either case, $h_{1}$ vanishes on a (countable) set with an accumulation point, and since $h_{1}$ is analytic it follows that $h_{1} \equiv 0$, a contradiction, and Claim 1 holds.

Now, to prove Claim 2, we follow the proof of Lemma 4.2 of [10]. For a fixed $\omega \in X_{4}$, define $f_{\omega}: \mathbb{C} \rightarrow \mathbb{C}$ by

$$
f_{\omega}(z)=\left\langle e_{z}, S_{\omega} \mathbb{1}\right\rangle=\int e^{2 \pi i z x} \overline{\left[S_{\omega} \mathbb{1}\right](x, y)} d\left(\mu_{4} \times \lambda\right)
$$

Since the distribution $\overline{\left[S_{\omega} \mathbb{1}\right](x, y)} d\left(\mu_{4} \times \lambda\right)$ is compactly supported, a standard convergence argument demonstrates that $f_{\omega}$ is entire. Likewise, $f_{\omega}^{*}(z)=\overline{f_{\omega}(\bar{z})}$ is entire, and for $t$ real,

$$
f_{\omega}(t) f_{\omega}^{*}(t)=\left(\left\langle e_{t}, S_{\omega} \mathbb{1}\right\rangle\right)\left(\overline{\left\langle e_{t}, S_{\omega} \mathbb{1}\right\rangle}\right)=\left|\left\langle e_{t}, S_{\omega} \mathbb{1}\right\rangle\right|^{2}
$$

Thus,

$$
h_{X}(t)=\sum_{\omega \in X_{4}} f_{\omega}(t) f_{\omega}^{*}(t)
$$

For $n \in \mathbb{N}$, let $h_{n}(z)=\sum_{|\omega| \leq n} f_{\omega}(z) f_{\omega}^{*}(z)$, which is entire. By Hölder's inequality,

$$
\begin{aligned}
\sum_{\omega \in X_{4}}\left|f_{\omega}(z) f_{\omega}^{*}(z)\right| & \leq\left(\sum_{\omega \in X_{4}}\left|\left\langle e_{z}, S_{\omega} \mathbb{1}\right\rangle\right|^{2}\right)^{1 / 2}\left(\sum_{\omega \in X_{4}}\left|\left\langle e_{\bar{z}}, S_{\omega} \mathbb{1}\right\rangle\right|^{2}\right)^{1 / 2} \\
& \leq\left\|e_{z}\right\|\left\|e_{\bar{z}}\right\| \\
& \leq e^{K \operatorname{Im}(z)}
\end{aligned}
$$

for some constant $K$. Thus, the sequence $h_{n}(z)$ converges pointwise to a function $h(z)$, and are uniformly bounded on strips $\operatorname{Im}(z) \leq C$. By the theorems of Montel and Vitali, the limit function $h$ is entire, which coincides with $h_{X}$ for real $t$, and Claim 2 is proved.

Example 1. As mentioned in Section 2, in general, $\left\{S_{\omega} \mathbb{1}\right\}$ need not be complete, and the exceptional point $\rho=-1$ in Theorem 2 provides the example. In the case $\rho=-1$, the set (6) becomes

$$
\left\{d_{n} e^{2 \pi i n x}: n \in \mathbb{N}_{0}\right\}
$$

where the coefficients $d_{n}=1$ if $n \in \Gamma_{3}$ and 0 otherwise. Here,

$$
\Gamma_{3}=\left\{\sum_{n=0}^{N} l_{n} 4^{n}: l_{n} \in\{0,3\}\right\}
$$

and it is known [4] that the sequence $\left\{e^{2 \pi i n x}: n \in \Gamma_{3}\right\}$ is incomplete in $L^{2}\left(\mu_{4}\right)$. Thus, $\left\{P_{V} S_{\omega} \mathbb{1}\right\}$ is incomplete in $V$, so $\left\{S_{\omega} \mathbb{1}\right\}$ is incomplete in $L^{2}\left(\mu_{4} \times \lambda\right)$.

We can generalize the construction of Theorem 2 as follows. We want to choose a matrix

$$
A=\left(\begin{array}{cccc}
\frac{1}{2} & \frac{1}{2} & \frac{1}{2} & \frac{1}{2} \\
h_{10} & h_{11} & h_{12} & h_{13} \\
h_{20} & h_{21} & h_{22} & h_{23} \\
h_{30} & h_{31} & h_{32} & h_{33}
\end{array}\right)
$$


such that $\left(\begin{array}{llll}1 & -1 & 1 & -1\end{array}\right)^{T}$ is in the kernel of $H$ and the matrix

$$
H=\left(\begin{array}{rrrr}
\frac{1}{2} & \frac{1}{2} & \frac{1}{2} & \frac{1}{2} \\
h_{10} & -h_{11} & h_{12} & -h_{13} \\
h_{20} & h_{21} & h_{22} & h_{23} \\
h_{30} & -h_{31} & h_{32} & -h_{33}
\end{array}\right)
$$

is unitary. We obtain a system of nonlinear equations in the 12 unknowns. To parametrize all solutions, we consider the following row vectors:

$$
\begin{aligned}
\vec{v}_{0} & =\frac{1}{2}\left(\begin{array}{llll}
1 & 1 & 1 & 1
\end{array}\right) & \vec{w}_{0} & =\frac{1}{2}\left(\begin{array}{lllll}
1 & -1 & 1 & -1
\end{array}\right) \\
\vec{v}_{1} & =\frac{1}{2}\left(\begin{array}{lllll}
1 & -1 & -1 & 1
\end{array}\right) & \vec{w}_{1} & =\frac{1}{2}\left(\begin{array}{llll}
1 & 1 & -1 & -1
\end{array}\right) \\
\vec{v}_{2} & =\frac{1}{2}\left(\begin{array}{llllll}
1 & 1 & -1 & -1
\end{array}\right) & \vec{w}_{2} & =\frac{1}{2}\left(\begin{array}{lllll}
1 & -1 & -1 & 1
\end{array}\right)
\end{aligned}
$$

If we construct the matrix $A$ so that the rows are linear combinations of $\left\{\vec{v}_{0}, \vec{v}_{1}, \vec{v}_{2}\right\}$, then $A$ will satisfy the desired condition on the kernel. Note that if the $j$-th row of $A$ is $\alpha_{j 0} \vec{v}_{0}+$ $\alpha_{j 1} \vec{v}_{1}+\alpha_{j 2} \vec{v}_{2}$ for $j=1,3$, then the $j$-th row of $H$ is $\alpha_{j 0} \vec{w}_{0}+\alpha_{j 1} \vec{w}_{1}+\alpha_{j 2} \vec{w}_{2}$, whereas if $j=0,2$, then the $j$-th row of $H$ is equal to the $j$-th row of $A$.

Thus, we want to choose coefficients $\alpha_{j k}, j=0,1,2,3, k=1,2,3$ so that the matrix

$$
H=\left(\begin{array}{c}
\alpha_{00} \vec{v}_{0}+\alpha_{01} \vec{v}_{1}+\alpha_{02} \vec{v}_{2} \\
\alpha_{10} \vec{w}_{0}+\alpha_{11} \vec{w}_{1}+\alpha_{12} \vec{w}_{2} \\
\alpha_{20} \vec{v}_{0}+\alpha_{21} \vec{v}_{1}+\alpha_{22} \vec{v}_{2} \\
\alpha_{30} \vec{w}_{0}+\alpha_{31} \vec{w}_{1}+\alpha_{32} \vec{w}_{2}
\end{array}\right)
$$

is unitary. To satisfy the requirement on the first row, we choose $\alpha_{00}=1$ and $\alpha_{01}=\alpha_{02}=0$. Calculating the inner products of the rows of $H$, we obtain the following necessary and sufficient conditions:

$$
\begin{aligned}
\left|\alpha_{j 0}\right|^{2}+\left|\alpha_{j 1}\right|^{2}+\left|\alpha_{j 2}\right|^{2} & =1 \\
\alpha_{00} \overline{\alpha_{20}} & =0 \\
\alpha_{11} \overline{\alpha_{22}}+\alpha_{12} \overline{\alpha_{21}} & =0 \\
\alpha_{10} \overline{\alpha_{30}}+\alpha_{11} \overline{\alpha_{31}}+\alpha_{12} \overline{\alpha_{32}} & =0 \\
\alpha_{21} \overline{\alpha_{32}}+\alpha_{22} \overline{\alpha_{31}} & =0
\end{aligned}
$$

Proposition 2. Fix $\alpha_{00}=1$. There exists a solution to the Equations (16) - (20) if and only if $\alpha_{10}, \alpha_{30} \in \mathbb{C}$ with

$$
\left|\alpha_{10}\right|^{2}+\left|\alpha_{30}\right|^{2}=1
$$

Proof. $(\Leftarrow)$ If $\left|\alpha_{10}\right|^{2}=1$, then we choose $\alpha_{21}=\alpha_{31}=1$ and all other coefficients to be 0 to obtain a solution to Equations (16) - (20). Likewise, if $\left|\alpha_{10}\right|^{2}=0$, then choose $\alpha_{11}=\alpha_{21}=1$ and all other coefficients to be 0 . 
Now suppose that $0<\left|\alpha_{10}\right|<1$, and we choose $\lambda=\frac{-\overline{\alpha_{10}} \alpha_{30}}{1-\left|\alpha_{10}\right|^{2}}$. Then choose $\alpha_{11}$ and $\alpha_{12}$ such that $\left|\alpha_{11}\right|^{2}+\left|\alpha_{12}\right|^{2}=1-\left|\alpha_{10}\right|^{2}$. Now let $\alpha_{31}=\lambda \alpha_{11}$ and $\alpha_{32}=\lambda \alpha_{12}$. We have

$$
\begin{aligned}
\alpha_{10} \overline{\alpha_{30}}+\alpha_{11} \overline{\alpha_{31}}+\alpha_{12} \overline{\alpha_{32}} & =\alpha_{10} \overline{\alpha_{30}}+\bar{\lambda}\left|\alpha_{11}\right|^{2}+\bar{\lambda}\left|\alpha_{12}\right|^{2} \\
& =\alpha_{10} \overline{\alpha_{30}}+\bar{\lambda}\left(1-\left|\alpha_{10}\right|^{2}\right) \\
& =0
\end{aligned}
$$

so Equation (19) is satisfied.

Equation (17) forces $\alpha_{20}=0$; choose $\alpha_{21}$ and $\alpha_{22}$ such that $\left|\alpha_{21}\right|^{2}+\left|\alpha_{22}\right|^{2}=1$ and $\alpha_{11} \overline{\alpha_{21}}+$ $\alpha_{12} \overline{\alpha_{22}}=0$. Thus, Equations (18) and (20) are satisfied. Finally, regarding Equation (16), it is satisfied for $j=0,1,2$ by construction. For $j=3$, we calculate:

$$
\begin{aligned}
\left|\alpha_{30}\right|^{2}+\left|\alpha_{31}\right|^{2}+\left|\alpha_{32}\right|^{2} & =\left|\alpha_{30}\right|^{2}+|\lambda|^{2}\left(\left|\alpha_{11}\right|^{2}+\left|\alpha_{12}\right|^{2}\right) \\
& =\left|\alpha_{30}\right|^{2}+\frac{\left|\alpha_{10}\right|^{2}\left|\alpha_{30}\right|^{2}}{\left(1-\left|\alpha_{10}\right|^{2}\right)^{2}}\left(1-\left|\alpha_{10}\right|^{2}\right) \\
& =\left|\alpha_{30}\right|^{2}\left(1+\frac{\left|\alpha_{10}\right|^{2}}{1-\left|\alpha_{10}\right|^{2}}\right) \\
& =\frac{\left|\alpha_{30}\right|^{2}}{1-\left|\alpha_{10}\right|^{2}} \\
& =1
\end{aligned}
$$

as required.

$(\Rightarrow)$ Suppose that we have a solution to Equations (16) - (20). If $\left|\alpha_{10}\right|=1$, then we must have $\alpha_{11}=\alpha_{12}=0$, and thus Equation (19) requires $\alpha_{30}=0$, so Equation (21) holds.

Now suppose $\left|\alpha_{10}\right|<1$. Since $\alpha_{20}=0$, we must have that $\left|\alpha_{21}\right|^{2}+\left|\alpha_{22}\right|^{2}=1$. Combining this with Equations (18) and (20) imply that the matrix

$$
\left(\begin{array}{ll}
\alpha_{11} & \alpha_{12} \\
\alpha_{31} & \alpha_{32}
\end{array}\right)
$$

is singular. Thus, there exists a $\lambda$ such that $\alpha_{31}=\lambda \alpha_{11}$ and $\alpha_{32}=\lambda \alpha_{12}$. Using the same computation as in Equation (22), we conclude that $\lambda=\frac{-\overline{\alpha_{10}} \alpha_{30}}{1-\left|\alpha_{10}\right|^{2}}$; then Equation (23) implies (21).

The coefficient matrix we obtain from this construction is

$$
H=\frac{1}{2}\left(\begin{array}{rrrr}
1 & 1 & 1 & 1 \\
\alpha_{10}+\alpha_{11}+\alpha_{12} & \alpha_{10}-\alpha_{11}+\alpha_{12} & \alpha_{10}-\alpha_{11}-\alpha_{12} & \alpha_{10}+\alpha_{11}-\alpha_{12} \\
\alpha_{21}+\alpha_{22} & -\alpha_{21}+\alpha_{22} & -\alpha_{21}-\alpha_{22} & \alpha_{21}-\alpha_{22} \\
\alpha_{30}+\lambda \alpha_{11}+\lambda \alpha_{12} & \alpha_{30}-\lambda \alpha_{11}+\lambda \alpha_{12} & \alpha_{30}-\lambda \alpha_{11}-\lambda \alpha_{12} & \alpha_{30}+\lambda \alpha_{11}-\lambda \alpha_{12}
\end{array}\right)
$$

where we are allowed to choose $\alpha_{11}, \alpha_{12}, \alpha_{21}$ and $\alpha_{22}$ subject to the normalization condition in Equation (16). However, those choices do not affect the construction, since if we apply Proposition 1 and the calculation from Theorem 2, we obtain

$$
P_{V} S_{\omega} \mathbb{1}=\left(\alpha_{10}\right)^{\ell_{1}(n)} \cdot(0)^{\left.\ell_{2}(n)\right)} \cdot\left(\alpha_{30}\right)^{\ell_{3}(n)} e^{2 \pi i n x} .
$$

This will in fact be a Parseval frame for $L^{2}\left(\mu_{4}\right)$, provided $V \subset \mathcal{K}$, as in the proof of Theorem 2 . 
Theorem 3. Suppose $p, q \in \mathbb{C}$ with $|p|^{2}+|q|^{2}=1$. Then

$$
\left\{p^{\ell_{1}(n)} \cdot 0^{\ell_{2}(n)} \cdot q^{\ell_{3}(n)} e^{2 \pi i n x}: n \in \mathbb{N}_{0}\right\}
$$

is a Parseval frame for $L^{2}\left(\mu_{4}\right)$, provided $p \neq 0$.

Proof. Substitute $\alpha_{10}=p$ and $\alpha_{30}=q$ in Proposition 2 and Equation (24). As noted, we only need to verify $V \subset \mathcal{K}$. We proceed as in the proof of Theorem 2 indeed, define $f, h_{X}, \mathfrak{m}_{j}$ and $g_{j}$ as previously. We obtain $b_{0}=1, b_{1}=\bar{p}, b_{2}=0$, and $b_{3}=\bar{q}$, so Equation (8) becomes

$$
h_{X}(t)=\cos ^{2}\left(\frac{\pi t}{2}\right) h_{X}\left(\frac{t}{4}\right)+|\bar{p}|^{2} \sin ^{2}\left(\frac{\pi t}{2}\right) h_{X}\left(\frac{t-1}{4}\right)+|\bar{q}|^{2} \sin ^{2}\left(\frac{\pi t}{2}\right) h_{X}\left(\frac{t-3}{4}\right) .
$$

From here, the same argument shows that $h_{X} \equiv 1$, and $V \subset \mathcal{K}$.

\section{Concluding Remarks}

We remark here that the constructions given above for $\mu_{4}$ does not work for $\mu_{3}$. Indeed, we have the following no-go result. To obtain the measure $\mu_{3} \times \lambda$, we consider the iterated function system:

$$
\Upsilon_{0}(x, y)=\left(\frac{x}{3}, \frac{y}{2}\right), \Upsilon_{1}(x, y)=\left(\frac{x+2}{3}, \frac{y}{2}\right), \Upsilon_{2}(x, y)=\left(\frac{x}{3}, \frac{y+1}{2}\right), \Upsilon_{3}(x, y)=\left(\frac{x+2}{3}, \frac{y+1}{2}\right) .
$$

Using the same choice of filters, the matrix $\mathcal{M}(x, y)$ reduces to

$$
H=\left(\begin{array}{rrrr}
a_{00} & a_{01} & a_{02} & a_{03} \\
a_{10} & e^{4 \pi i / 3} a_{11} & a_{12} & e^{4 \pi i / 3} a_{13} \\
a_{20} & e^{2 \pi i / 3} a_{21} & a_{22} & e^{2 \pi i / 3} a_{23} \\
a_{30} & a_{31} & a_{32} & a_{33}
\end{array}\right)
$$

which we require to be unitary. Additionally, we require the same conditions as for $\mu_{4}$, namely, the first row of $H$ must have all entries $\frac{1}{2}$, and $a_{j 0}+a_{j 2}=a_{j 1}+a_{j 3}$. The inner product of the first two rows must be 0 . Hence,

$$
\frac{1}{2}\left(a_{10}+e^{4 \pi i / 3} a_{11}+a_{12}+e^{4 \pi i / 3} a_{13}\right)=\frac{1}{2}\left(a_{10}+a_{12}\right)\left(1+e^{4 \pi i / 3}\right)=0 .
$$

Consequently, $a_{10}+a_{12}=0$. Likewise, $a_{20}+a_{22}=a_{30}+a_{32}=0$. As a result,

$$
H\left(\begin{array}{l}
1 \\
0 \\
1 \\
0
\end{array}\right)=\left(\begin{array}{l}
a_{00}+a_{02} \\
a_{10}+a_{12} \\
a_{20}+a_{22} \\
a_{30}+a_{32}
\end{array}\right)=\left(\begin{array}{l}
1 \\
0 \\
0 \\
0
\end{array}\right)
$$

and so $H$ cannot be unitary.

It may be possible to extend the construction for $\mu_{4}$ to $\mu_{3}$ by considering a representation of $\mathcal{O}_{n}$ for some sufficiently large $n$, or by considering $\mu_{3} \times \rho$ for some other fractal measure $\rho$ rather than $\lambda$.

Acknowledgement. We thank Dorin Dutkay for assisting with the proof of Claim 1 in Theorem 2. 


\section{REFERENCES}

1. Akram Aldroubi, Portraits of frames, Proc. Amer. Math. Soc. 123 (1995), no. 6, 1661-1668. MR 1242070 (95g:46037)

2. Xin-Rong Dai, Xing-Gang He, and Chun-Kit Lai, Spectral property of Cantor measures with consecutive digits, Adv. Math. 242 (2013), 187-208. MR 3055992

3. R. J. Duffin and A. C. Schaeffer, A class of nonharmonic Fourier series, Trans. Amer. Math. Soc. 72 (1952), 341-366. MR 0047179 (13,839a)

4. Dorin Ervin Dutkay, Deguang Han, and Qiyu Sun, On the spectra of a Cantor measure, Adv. Math. 221 (2009), no. 1, 251-276. MR 2509326 (2010f:28013)

5. Dorin Ervin Dutkay, Deguang Han, and Eric Weber, Bessel sequences of exponentials on fractal measures, J. Funct. Anal. 261 (2011), no. 9, 2529-2539. MR 2826404 (2012h:42059)

6. Continuous and discrete Fourier frames for fractal measures, Trans. Amer. Math. Soc. 366 (2014), no. 3, 1213-1235. MR 3145729

7. Dorin Ervin Dutkay, Gabriel Picioroaga, and Myung-Sin Song, Orthonormal bases generated by Cuntz algebras, J. Math. Anal. Appl. 409 (2014), no. 2, 1128-1139. MR 3103223

8. Deguang Han and David R. Larson, Frames, bases and group representations, Mem. Amer. Math. Soc. 147 (2000), no. 697, x+94. MR 1686653 (2001a:47013)

9. John E. Hutchinson, Fractals and self-similarity, Indiana Univ. Math. J. 30 (1981), no. 5, 713-747. MR MR625600 (82h:49026)

10. Palle E. T. Jorgensen and Steen Pedersen, Dense analytic subspaces in fractal $L^{2}$-spaces, J. Anal. Math. 75 (1998), 185-228. MR MR1655831 (2000a:46045)

11. Izabella Łaba and Yang Wang, On spectral Cantor measures, J. Funct. Anal. 193 (2002), no. 2, 409-420. MR 1929508 (2003g:28017)

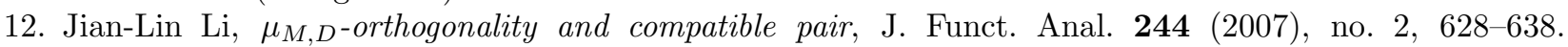
MR 2297038 (2008h:42046)

13. Joaquim Ortega-Cerdà and Kristian Seip, Fourier frames, Ann. of Math. (2) 155 (2002), no. 3, 789-806. MR MR1923965 (2003k:42055)

14. Robert S. Strichartz, Mock Fourier series and transforms associated with certain Cantor measures, J. Anal. Math. 81 (2000), 209-238. MR 1785282 (2001i:42009)

Department of Mathematical Sciences, 414 E. Clark St., University of South Dakota, VERMILLION, SD 57069

E-mail address: gabriel.picioroaga@usd.edu

Department of Mathematics, Iowa State University, 396 Carver Hall, Ames, IA 50011

E-mail address: esweber@iastate.edu 